\title{
DESIGN AND IMPLEMENTATION OF MICROWAVE PLANNING TOOL WITH STUDYING THE EFFECT OF VARIOUS ASPECTS
}

\author{
Mohamed Maher Hanafi, Mohamed Hamza EL-Saba, and Hussein A.Elsayed \\ Electronics and Communication Eng. Dept, Ain Shams University
}

\begin{abstract}
Microwave Line-of-sight radio is one of most important and common transmission methods in telecommunications networks. As the microwave radio signals are propagated through the lower atmosphere, they are sensitive to terrain, atmospheric, and climatic conditions. The planning and design of a reliable microwave links is very difficult and require a lot of complex computations. Therefore, not only software implementation is required, but studding the effect of all the different design aspects is very crucial for the telecommunication systems. The software then estimates the path profile, link budget, fade margin, and all other parameters at any place.

This paper presents the microwave software planning tool design and implementation with study of all of the design parameters. This software is an engineering tool to aid in the design and planning of the microwave transmission links considering the geography, distance, antenna height, transmit power, frequency, temperature, atmospheric effect, pressure, losses, and other factors which affects on the microwave line-of-sight radio link
\end{abstract}

\section{INTRODUCTION}

Microwave radio transmission is the transmission of information by electromagnetic waves with very small wavelengths. A microwave link is a communications system that uses a beam of microwave radio to transmit information between two locations, which ranges from just a few meters to several kilometers. There are some programs that can configure network, estimate path profile to find antenna height in each station, calculate link budget, receiver level, and link availability for microwave sites. This study is an attempt to design and implement microwave transmission planning tool to enable telecommunication engineers to design the microwave transmission sites based on geography, distance, antenna height, transmit power, frequency, temperature, water vapor, pressure, losses, and other factors to create the best microwave line-of-sight radio link. The tool also connects to online maps servers to draw the path profile and import it into the tool when user creates a new link. It uses the terrain curve and mathematical equations to simulate very accurate radio transmission link between any two sites. In the implemented microwave planning tool, users can add extra obstacles manually depending on the site survey, then the "path profile chart" page will display if there is a clear line-of-sight or not. The implemented tool doesn't required setup so it allows users to easily and quickly design the microwave transmission networks.

This software is designed to support equipment manufacturers, telecommunications, coordination, and engineering service providers worldwide. It has been taken into account that this software may be used by civil engineers and in site acquisition departments in the mobile service providers. Therefore, the technical idioms in the input parameters and the output results are reduced.

\section{Microwave Propagation and Free SPaCe Propagation}

The microwave beam is an electromagnetic wave that propagates in free space as well as material substances. The electromagnetic wave consists of two fields: electric field and magnetic field. In free space they are in phase and mutually perpendicular. If the microwave signal traveled in a vacuum, the 
characteristics of the microwave systems are determined by the mechanisms affecting the propagation of radio waves. These characteristics depend on the frequencies used for transmission, the atmospheric effects, and the earth terrain. The received signal can therefore be the resultant of any number of the following ways as shown in Fig. 1:

a) Direct waves (free-space).

b) Reflected waves (reflected from the ground).

c) Sky waves (reflected from ionized layers above the earth and is known as the ionosphere).

d) Surface waves (caused by diffraction around the earth).

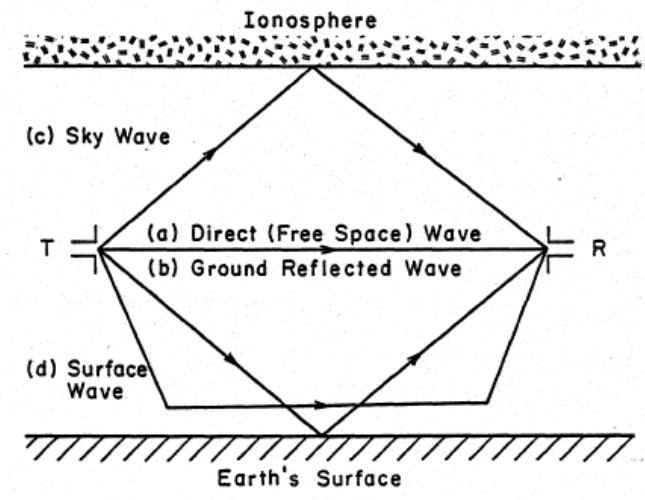

Fig. 1. Transmission paths between the transmitter and receiver.

Microwave radio transmission requires a line-of-sight path between transmitting and receiving antennas. Free space path loss is the loss in signal strength of an electromagnetic wave that would result from a line-of-sight path through free space, it occurs when both transmitting and receiving antennas are located away from the influence of the Earth's surface or other reflecting and absorbing objects. These conditions provide a highly predictable and stable power loss and are considered ideal conditions. Free space loss is expressed as the ratio of the power into an isotropic transmitting antenna to the power output from an isotropic receiving antenna. The Free space loss is given by equation (1).

$$
F S L=\left(\frac{4 \pi \mathrm{d}}{\lambda}\right)^{2}=\left(\frac{4 \pi \mathrm{d} f}{c}\right)^{2}
$$

where $\lambda$ is free space wavelength, $\mathrm{d}$ is the distance between two endpoints is measured in $\mathrm{km}$, and the frequency $\mathrm{f}$ in $\mathrm{GHz}$. For simplicity, the path loss equation can be expressed as in equation (2).

$$
\mathrm{FSL}(\mathrm{dB})=20 \log _{10}(\mathrm{~d})+20 \log _{10}(\mathrm{f})+92.45
$$

\section{Path Profile and Fresnel Zone}

The radio path profile is a plot of the earth's elevations against the distance between the two endpoints that represent the microwave path. For long distance, map work analysis is essential because physically checking for the Line-of-sight is impossible. The preferred method of doing so is to produce a path profile which is a cross-section of the Earth's surface between the two end points. Digital map is required and the contour line elevations recorded from a straight line are drawn between the two points on the map. The Earth's bulge and the curvature of the radio beam need to be taken into account. Critical obstruction points identified on the path profile should always be physically inspected for additional obstructions such as trees or buildings. The Earth bulge is determined as in equation (3).

$$
b=d_{1} \cdot d_{2} / 12.75 k
$$

where $\mathrm{b}$ is in meters, $\mathrm{d} 1, \mathrm{~d} 2$ are the distances from each end site to a certain point in kilometers, and $\mathrm{k}$ is the effective Earth radius factor. The microwave beam between two endpoints is not a straight line but a wave front has a cross-sectional width of which the direct ray is the axis. There are a series of concentric ellipsoidal regions; the measure of beam width on one of them is the first Fresnel zone, which is an ellipsoid containing most of the signal power that reaches the receiving antenna as shown in Figure 2. The clearance required along the path in order not to interfere this wave front along the path. This clearance is a function of the distance between the two end points and the frequency of operation

For a fixed path, the first Fresnel zone becomes narrower with increasing frequency and larger antennas. Path profiles show the clearance of a microwave beam and its Fresnel zones above the ground 
with the effective Earth radius factor as the parameter. The required clearance creates a cigar shape between the endpoints and it described by 0.6 of the first Fresnel zone.

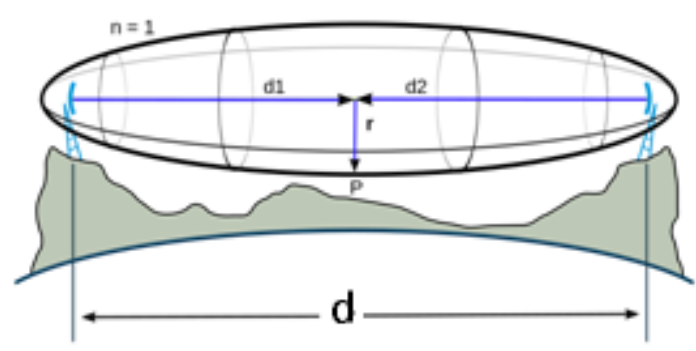

Figure 2 Fresnel Zone

The general equation for calculating the Fresnel zone radius at any point $\mathrm{P}$ in between the endpoints of the link is shown in equation (4):

$$
F=17.32 \sqrt{\frac{d_{1} * d_{2}}{f d}}
$$

Where $F$ is the radius of the Fresnel Zone in meter, $d_{1}$ is the distance to $P$ from one end in $K m, d_{2}$ is the distance from $\mathrm{P}$ to the other end in $\mathrm{Km}, \mathrm{f}$ is the operation frequency in $\mathrm{GHz}$ and $\mathrm{d}$ is the total distance between two endpoints.

\section{Multipath FAding}

Multipath fading is the drop in the received signal due to phase cancellation between the direct path signal and one or more signals traveling over different paths. The multipath cancellation is caused by reflection or refraction of the microwave beam. The reflection occurs when a portion of the wave front is reflected by the surface of the earth and arrives out of phase with the direct signal. The outages due to multipath fading function of parameters such as frequency, hop length, terrain type and roughness, climatic conditions, path clearance and geoclimatic factor determined from the hop terrain and climatic zone.

Each country has unique parameters; some of these parameters can be obtained by following the various versions of ITU 530. In fact, multipath fading causes a fast and deep signal attenuation that can cause an outage if the fade margin is exceeded. So, when designing a radio link, it is important to know the probability of this event occurring relative to the depth of fading. The formulas and methods presented by ITU are an attempt to define prediction models that allow you to accurately predict the outage time for any given hop. The probability distribution curve follows a Rayleigh distribution [2] for deep fades; the Rayleigh fading is given as:

$$
p(F<M)=P_{o} * 10^{-M / 10}
$$

That mean the probability of a fade exceeding a set fade margin $\mathrm{M}$ is proportional to a set multipath fading occurrence factor Po. Because the fading is caused by multipath, one tries to predict the probability of multiple paths existing. The equation for calculating the geoclimatic factor $\mathrm{K}$ [4] is as follows:

$$
K=5.0 * 10^{-7} * 10^{-0.1\left(C_{o}-C_{L a t}-C_{L o n}\right)} P L^{1.5}
$$

where $C_{\mathrm{o}}$ is the terrain altitude coefficient [4] and takes the values

$$
C_{0}= \begin{cases}1.7 & \text { from } 0-400 \mathrm{~m} \text { AMSL } \\ 4.2 & \text { from } 400 \mathrm{~m}-700 \mathrm{~m} \\ 8 & \text { above } 700 \mathrm{~m}\end{cases}
$$

the coefficient $C_{\text {Lat }}$ of latitude $\xi$ [4] is given by: 


$$
\begin{array}{lll}
C_{\text {Lat }}=0 & \text { (dB) } & \text { for } \xi \leq 53^{\circ} \mathrm{N} \text { or }{ }^{\circ} \mathrm{S} \\
C_{\text {Lat }}=-53+\xi & \text { (dB) } & \text { for } 53^{\circ} \mathrm{N} \text { or }{ }^{\circ} \mathrm{S}<\xi<60^{\circ} \mathrm{N} \text { or }{ }^{\circ} \mathrm{S} \\
\mathrm{C}_{\text {Lat }}=7 & \text { (dB) } & \text { for } \xi \geq 60^{\circ} \mathrm{N} \text { or }{ }^{\circ} \mathrm{S}
\end{array}
$$

and the longitude coefficient $\mathrm{C}_{\mathrm{Lon}}$, [4] by:

$$
\begin{array}{lll}
C_{\text {Lon }}=3 & \text { (dB) } & \text { for longitudes of Europe and Africa } \\
C_{\text {Lon }}=-3 & \text { (dB) } & \text { for longitudes of North and South America } \\
C_{\text {Lon }}=0 & \text { (dB) } & \text { for all other longitudes }
\end{array}
$$

The equation for calculating the path inclination [5] is as follows:

$$
\epsilon_{P}=\left|h_{r}-h_{e}\right| / d
$$

Where $h_{r}$ and $h_{e}$ are the heights of the transmitting and receiving antennas above sea level and $d$ is the distance between two endpoints in kilometers. The average worst month fade probability [2], $\mathrm{P}_{\mathrm{w}}$ can thus be expressed as:

$$
P_{w}=K * d^{3.6} * f^{0.89}\left(1+\epsilon_{p}\right)^{-1.4} * 10^{-A / 10} \%
$$

Where $\mathrm{K}$ is the geoclimatic factor, $\mathrm{d}$ is the path length in kilometers, $\mathrm{f}$ is the frequency in gigahertz, $\epsilon_{\mathrm{p}}$ is the path inclination in milliard (max value 24 ), and $\mathrm{A}$ is the fade margin in decibels.

\section{ATMOSPHERIC ABSORPTION AND RAIN EFFECT}

Due to gases (especially oxygen) and water vapor along the microwave propagation path, a loss in microwave energy occurs. This loss is known as atmospheric attenuation. Most of this lost energy is normally absorbed by gases and water vapor and transformed into heat. The total atmospheric attenuation is the sum of the atmospheric absorption due to oxygen and the atmospheric absorption due to water vapor [2] and is expressed as follows:

$$
A_{a}=\left(\gamma_{\text {water }}+\gamma_{\text {oxygen }}\right)
$$

The atmospheric absorption due to oxygen is given by the Van Vleck equation [6] as follows:

$$
\gamma_{o}=\left[0.4909 \frac{P^{2}}{T^{5 / 2}} V_{1}\right]\left\{\frac{1}{1+2.904 * 10^{-4} \lambda^{2} p^{2} T^{-1} V_{1}^{2}}\right\}\left\{1+\frac{0.5 V_{2}}{\lambda^{2} V_{1}}\right\}
$$

where: $\gamma_{0}$ is the atmospheric absorption due to Oxygen $\mathrm{dB} / \mathrm{km}, \mathrm{P}$ is the atmospheric pressure in millibars, $\mathrm{T}$ is the atmospheric temperature in Kelvin, $\lambda$ is the wavelength, $\mathrm{V}_{1}$ and $\mathrm{V}_{2}$ constants where $\mathrm{V}_{1}=0.018 \mathrm{~cm}^{-1}, \mathrm{~V}_{2}=0.05 \mathrm{~cm}^{-2}$. The atmospheric absorption due to water vapor from Van Vleck equation [6] is given by:

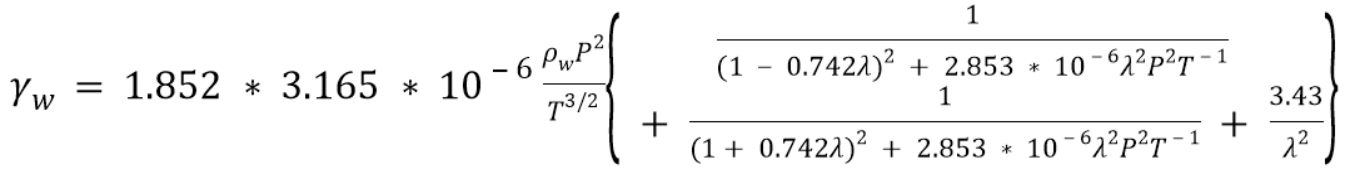

where $\gamma_{\mathrm{w}}$ is the water vapor density in $\mathrm{m}^{-3}$, Note that the atmospheric temperature for altitude less than $12 \mathrm{~km}$ is: $\mathrm{T}=188-6.5 \mathrm{~h}$, where $\mathrm{h}$ is the altitude in $\mathrm{km}$. Assuming that air pressure at sea level is 1015 millibars, then the air pressure in millibars at any altitude for up to $12 \mathrm{~km} \mathrm{[6]} \mathrm{is} \mathrm{given} \mathrm{by} \mathrm{:}$

$$
P=1015(1-0.02257 h)^{5.2561}
$$

To calculate the total Atmospheric absorption along the microwave propagation path [4] the equation will be as follows: 


$$
A_{a}=\left(\gamma_{\text {water }}+\gamma_{\text {oxygen }}\right) * \mathrm{~d}
$$

Where $\mathrm{d}$ is the distance between two endpoints.

The rain causes a degradation in the receive signal, this degradation is directly proportional to the frequency of the signal. Each particular raindrop contributes to the attenuation of the signal. The actual amount of fading is dependent on the frequency of the signal and the size of the raindrop. The two main causes of rain fading are scattering and absorption. Suitable statistical models are needed to relate the number of raindrops in a rain cell and their size distribution to the rain intensity. These models have been designed on the basis of a large amount of experimental data, coming from different regions in the world. Rain does not occur all times of year and its rate does not remain same all the time when it occurs. An important input to any rain attenuation model is the expected rain activity in the region where the radio hop will operate, as derived from long-term statistics. The rain rate exceeded for $0.01 \%$ of the time (in order to achieve $99.999 \%$ availability, for a given path length) is the significant parameter, useful to characterize the rainfall activity in a given region. The ITU-R recommendations can be used. In the last release of Rec. P-837 [13] a new approach is reported to estimate the rain rate exceeded for any percentage of time, in any part of the world. This is based on data files (available from the ITU website). These recommendations designed based on world maps with rain regions, Figure 3, each region was labeled with a letter, and each letter is associated with the corresponding rain rate in $\mathrm{mm} / \mathrm{h}$ as shown in Table 1 .

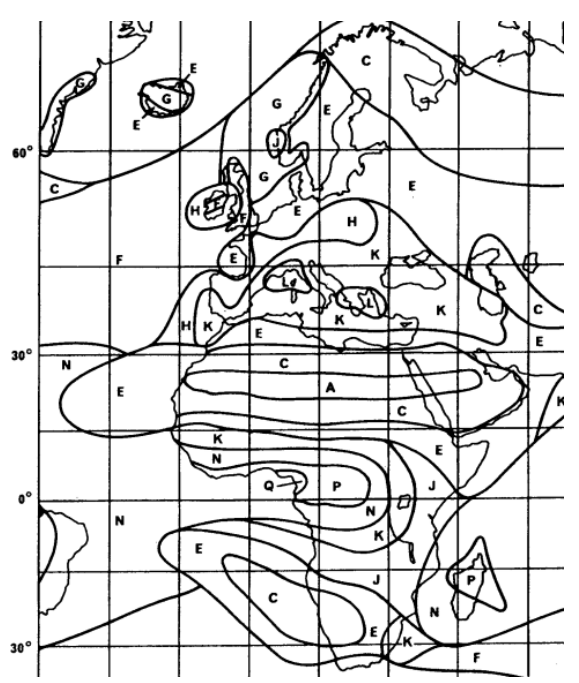

Figure 3 Example for maps with rain regions [13]

Table 1 The rain rate for every rain region according to the ITU-R recommendations.[13]

\begin{tabular}{|c|c|c|c|c|c|c|c|c|c|c|c|c|c|c|c|}
\hline $\begin{array}{c}\text { Peccentage } \\
\text { of time } \\
(\%)\end{array}$ & A & B & C & D & E & F & G & H & J & K & L & M & N & P & Q \\
\hline 1.0 & $<0.1$ & 0.5 & 0.7 & 21 & 0.6 & 1.7 & 3 & 2 & 8 & 15 & 2 & 4 & 5 & 12 & 24 \\
\hline 0.3 & 0.8 & 2 & 28 & 4.5 & 24 & 45 & 7 & 4 & 13 & 42 & 7 & 11 & 15 & 34 & 49 \\
\hline 0.1 & 2 & 3 & 5 & 8 & 6 & 8 & 12 & 10 & 20 & 12 & 15 & 22 & 35 & 65 & 72 \\
\hline 0.03 & 5 & 6 & 9 & 13 & 12 & 15 & 20 & 18 & 28 & 23 & 33 & 40 & 65 & 105 & 96 \\
\hline 0.01 & 8 & 12 & 15 & 19 & 22 & 28 & 30 & 32 & 35 & 42 & 60 & 63 & 95 & 145 & 115 \\
\hline 0.003 & 14 & 21 & 26 & 29 & 41 & 54 & 45 & 55 & 45 & 70 & 105 & 95 & 140 & 200 & 142 \\
\hline 0.001 & 22 & 32 & 42 & 42 & 70 & 78 & 65 & 83 & 55 & 100 & 150 & 120 & 180 & 250 & 170 \\
\hline
\end{tabular}

The specific rain attenuation is the parameter that gives the attenuation that the microwave link facing in each kilometer. The specific rain attenuation $\gamma_{R}(\mathrm{~dB} / \mathrm{km})$ can be expressed as a function of the rain rate $\mathrm{R}$ (in $\mathrm{mm} / \mathrm{h}$ ) [7] by the following exponential formula:

$$
\gamma_{R=k R^{\alpha}}
$$


where the parameters $\mathrm{k}$ and $\alpha$ are functions of the signal wavelength and polarization. ITU-R Rec. P838 [14] gives a table with the $\mathrm{k}$ and $\alpha$ value, for Vertical and Horizontal polarizations, in the frequency range 1 to $400 \mathrm{GHz}$ Table 1 .

Table 2 the ITU-R Rec. P-838 standards for the $k$ and $\alpha$ value, for Vertical and Horizontal polarizations, in the frequency range 1 to $40 \mathrm{GHz}$ [14].

\begin{tabular}{|c|c|c|c|c|}
\hline \multirow{2}{*}{$\begin{array}{l}\text { Frequency } \\
\mathrm{GHz}\end{array}$} & \multicolumn{2}{|c|}{ Horizontal polarisation } & \multicolumn{2}{|c|}{ Vertical polarisation } \\
\hline & $\mathrm{K}$ & $\alpha$ & $\mathrm{K}$ & $\alpha$ \\
\hline 1 & 0.0000387 & 0.912 & 0.0000352 & 0.880 \\
\hline 2 & 0.000154 & 0.963 & 0.000138 & 0.923 \\
\hline 4 & 0.000650 & 1.121 & 0.000591 & 1.075 \\
\hline 6 & 0.00175 & 1.308 & 0.00155 & 1.265 \\
\hline 7 & 0.00301 & 1.332 & 0.00265 & 1.312 \\
\hline 8 & 0.00454 & 1.327 & 0.00395 & 1.310 \\
\hline 10 & 0.0101 & 1.276 & 0.00887 & 1.264 \\
\hline 12 & 0.0188 & 1.217 & 0.0168 & 1.200 \\
\hline 15 & 0.0367 & 1.154 & 0.0335 & 1.128 \\
\hline 20 & 0.0751 & 1.099 & 0.0601 & 1.065 \\
\hline 25 & 0.124 & 1.061 & 0.113 & 1.030 \\
\hline 30 & 0.187 & 1.021 & 0.167 & 1.000 \\
\hline 35 & 0.263 & 0.979 & 0.233 & 0.963 \\
\hline 40 & 0.350 & 0.939 & 0.310 & 0.929 \\
\hline
\end{tabular}

The specific rain attenuation is not sufficient for computing the attenuation of the whole path, because there is a considerable temporal and spatial variation of the rain rate across the link. So, just multiplying the specific attenuation by the actual link path cannot properly give whole path attenuation. Instead, two new parameters are needed: rain cell length $d_{o}$ which mean the length over which the rain is considered as uniform, and effective path length $\mathrm{d}_{\text {eff }}$, which mean the average length of the intersection between cell and link. So, to calculate the effective path length [7], the equation will be as following:

$$
d_{e f f}=\frac{d}{1+\frac{d}{d_{o}}}
$$

Where $\mathrm{d}$ is the actual length of the terrestrial microwave link, and:

$$
\begin{aligned}
& \mathrm{R}_{0.01} \leq 100 \mathrm{~mm} / \mathrm{h} \\
& d_{o}=35 e^{-0.015 R_{0.01}} \\
& \text { And for } \mathrm{R}_{0.01}>100 \mathrm{~mm} / \mathrm{h} \\
& d_{o}=35 e^{-1.5}
\end{aligned}
$$

So, an estimate of the path attenuation (required fade margin due to rain) exceeded for $0.01 \%$ of the time [8] is given by:

$$
A_{0.01}=\gamma_{R} d_{e f f}
$$

Note that the rain unavailability is predicted as the probability that rain attenuation exceeds the Fade Margin that computed as a result of Link Budget calculation.

\section{LINK BUdGet AND FADE MARgin}

A link budget is accounting of all of the gains and losses from the transmitter, through the medium and to the receiver in the microwave radio transmission system which includes the transmitter antenna gain, the receiver antenna gain, the free-space path loss, and any additional losses caused by equipments, waveguides, cables, connectors, etc. Link Budget [2] which is called also Received Signal Level (RSL) equation will be as following:

$$
\mathrm{P}_{\mathrm{RX}}=\mathrm{P}_{\mathrm{TX}}+\mathrm{G}_{\mathrm{TX}}-\mathrm{L}_{\mathrm{TX}}-\mathrm{FSL}+\mathrm{G}_{\mathrm{RX}}-\mathrm{L}_{\mathrm{RX}}
$$

where $P_{R X}$ is the received signal power $(d B m), P_{T X}$ is the transmitter output power $(d B m), G_{T X}$ is the transmitter antenna gain $(\mathrm{dBi}), \mathrm{L}_{\mathrm{TX}}$ is the transmitter losses $(\mathrm{dB}), \mathrm{FSL}$ is the free space loss $(\mathrm{dB}), \mathrm{G}_{\mathrm{RX}}$ is the receiver antenna gain $(\mathrm{dBi}), \mathrm{L}_{\mathrm{RX}}$ is the receiver losses $(\mathrm{dB})$. 
The Fade Margin is the difference between the received signal level and the receiver threshold level. It is calculated to use it as a safety margin against fading that may occurs. In other words, it defined as the amount by which a received signal level may be reduced without causing system performance to fall below a specified threshold value.

7. FLOWCHART AND BLOCK DIAGRAM OF THE IMPLEMENTED MICROWAVE PLANNING TOOL Figure 4 and Error! Reference source not found. show the steps that the software follows to design a new line-of-sight radio link. It shows the scenario that the tool flows to collect data from the user, then processing it according to integrated equations, draw path profile, and then provide the user with the results.

At start up, the tool has to check the internet connectivity to get the geographical information from Google API according to the values that have stored from the last use. Next step the tool requires some needed information that set by the user such as: coordinates, Frequency, Antennas diameter, Polarization, Radio type and Tx power. Then, the tool has to check the internet connectivity again. If it is connected, it requests the geographical information from Google API and it draws the Map and the elevation path profile according to the new coordinates that set by the user. After that, the tool requests information such as: the elevation of earth above sea level at sites A and B, K-Factor, antenna height at sites $\mathrm{A} \& \mathrm{~B}$, and if there are any extra obstacles along the path. According to that information and if there is internet connectivity, the tool draws the path profile chart. The tool asks for $\mathrm{d} 1$ to calculate the first Fresnel zone. Then the tool asks for the some extra information such as: climate factor, terrain factor, terrain type, geographical region, latitude range, rain region, feeder losses, temperature, water vapor, and pressure. According to all above information, the tool calculates and displays the results. The results is: free space loss, flat fade margin, multipath fading losses, link availability, percentage of link down, atmospheric absorption, required fade margin against rain, specific attenuation due to rain and unavailability percentage due to rain.

The block diagram of the implemented microwave planning tool shows the relations between the equations and input and output parameters. It shows how the tool been built and describe how it relate all parameters together as shown in Figure 5. 

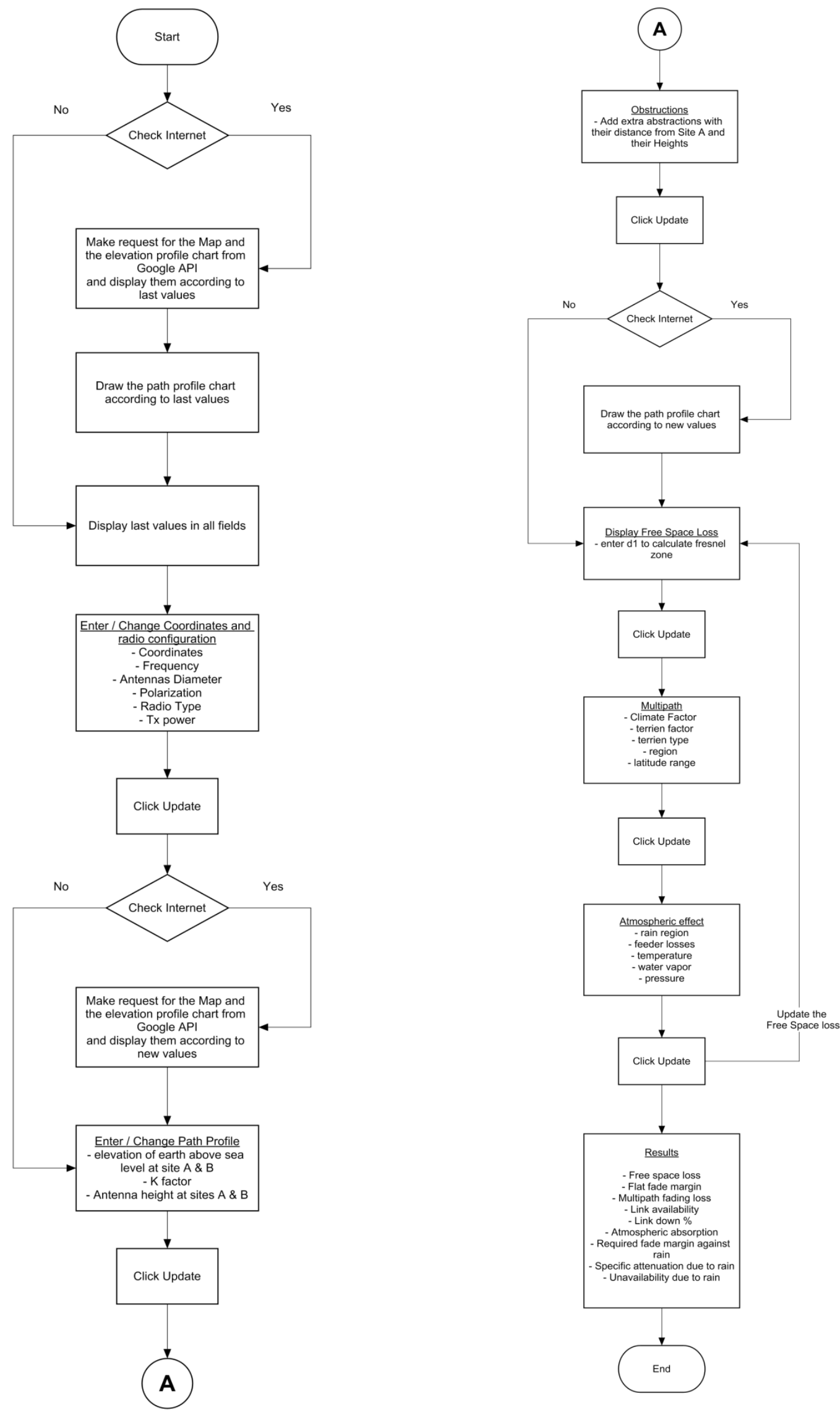

Figure 4 Flowchart of the implemented tool 


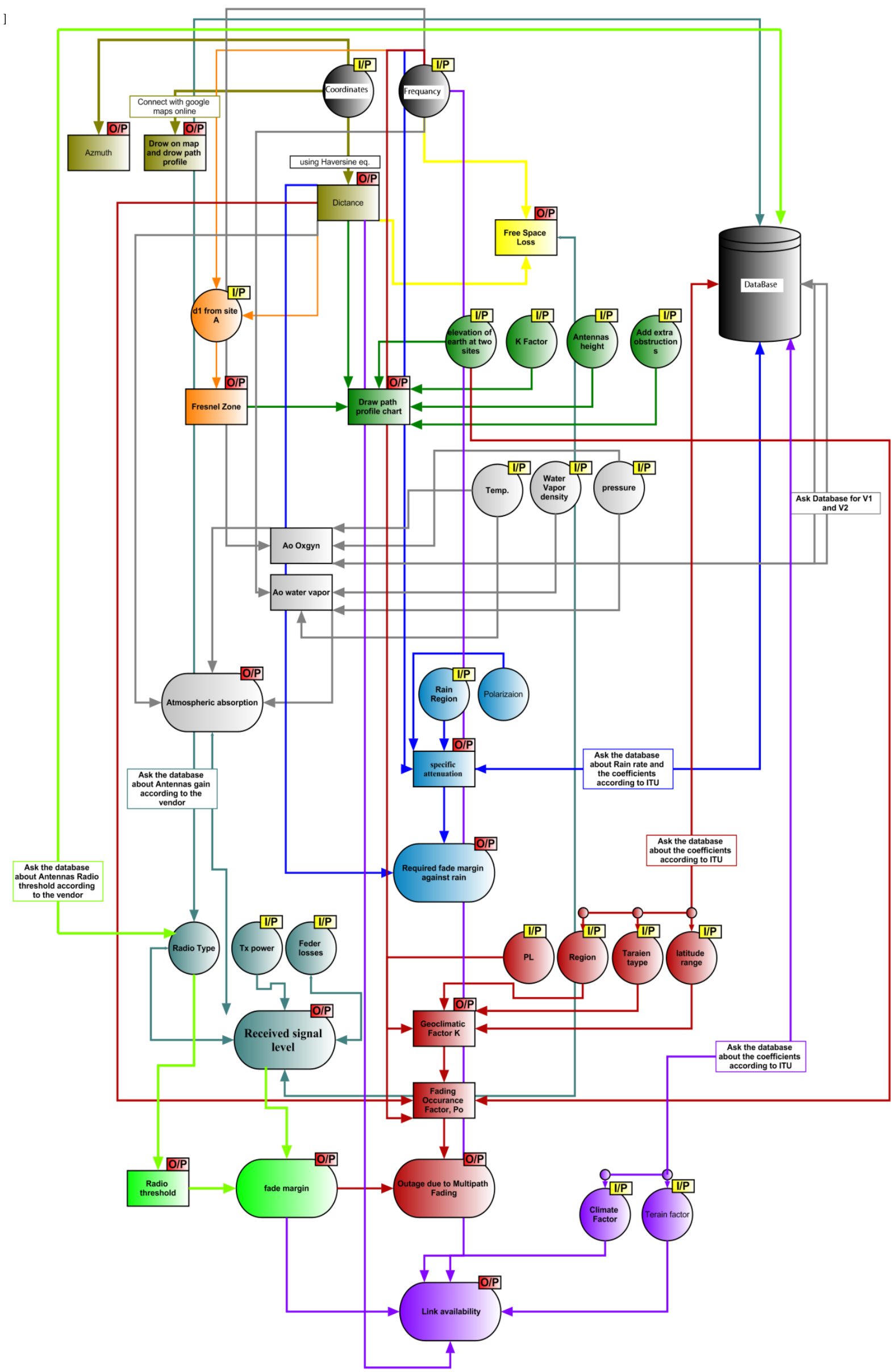

Figure 5 Block diagram of the implemented microwave planning tool. 


\section{SIMULATION RESULTS FOR THE IMPLEMENTED MICROWAVE PLANNING TOOL}

The implemented microwave planning tool is software developed to automatically design microwave point-to-point links. This software has nine parts: Coordinates and radio configuration, Map, Path profile, Obstructions, Path profile chart, Free space loss, Multipath, Atmospheric effect and Results. The following subsections explain those parts.

1) Coordinates and radio configuration:

In this part, the user has to add the coordinates and the radio configuration parameters as follows:

Area has been chosen: Sinai

Coordinates -can be obtained using GPS- :

Site A:

- Latitude: $27^{\circ} 48^{\prime} 42.2640 " \mathrm{~N}$

- Longitude: $33^{\circ} 34^{\prime} 16.7520^{\prime \prime} \mathrm{E}$

Site B:

- Latitude: $27^{\circ} 42^{\prime} 52.9920^{\prime \prime} \mathrm{N}$

- Longitude: $33^{\circ} 30^{\prime} 2.9880 " \mathrm{E}$

The operation Frequency selected: $7.617 \mathrm{GHz}$

The radio equipment selected: SIEMENS SRAL XD

Antenna A diameter: $1.2 \mathrm{~m}$

Antenna B diameter: $1.2 \mathrm{~m}$

Polarization: Vertical

Tx Power: $29 \mathrm{dBm}$

As such, the software will automatically convert the coordinates to the decimal form; it will calculate the distance between site A and B by using Haversine Formula. it will calculate the Azimuth from site A to B, and From B to A as shown in Fig. .

Fig. 6. Coordinates and radio configuration.

2) Map:

In this part, the software finds the location of the two sites A \& B and draw the direct path between them. It also draws the path profile (the elevation profile) for the direct path between site A \& B as shown in Fig. .

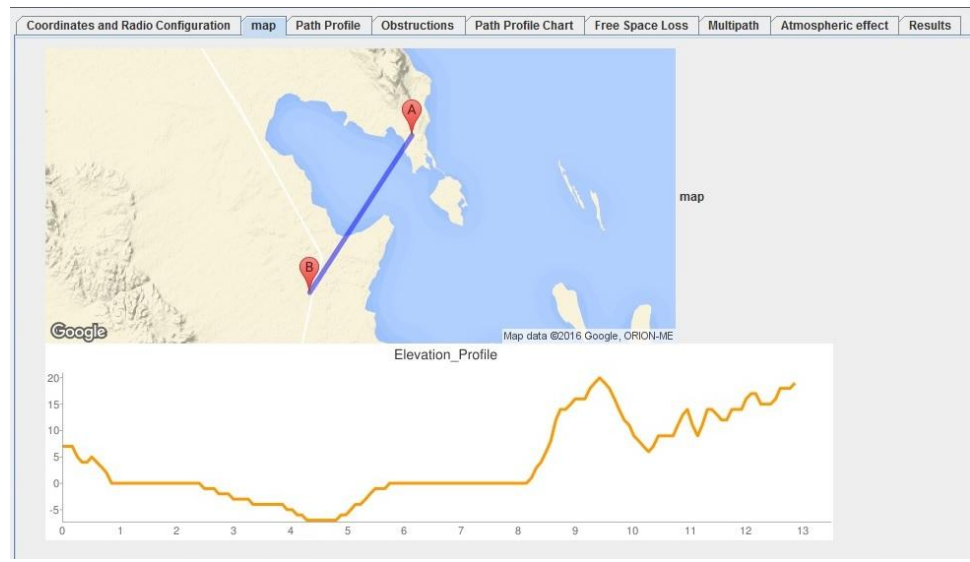

Fig. 7. Map.

3) Path profile:

In this part, the user defines the elevation of the earth above sea level at site $A \& B$, selects the curvature of the earth (the effective Earth radius factor), and defines the antennas height for site A \& B as shown in Figure. In the introduced case study, the elevation is taken as

- At site A: $5.5 \mathrm{~m}$

- At site B: $17.0 \mathrm{~m}$

and the antennas height as

- At site A: $30 \mathrm{~m}$

- At site B: $35 \mathrm{~m}$ 


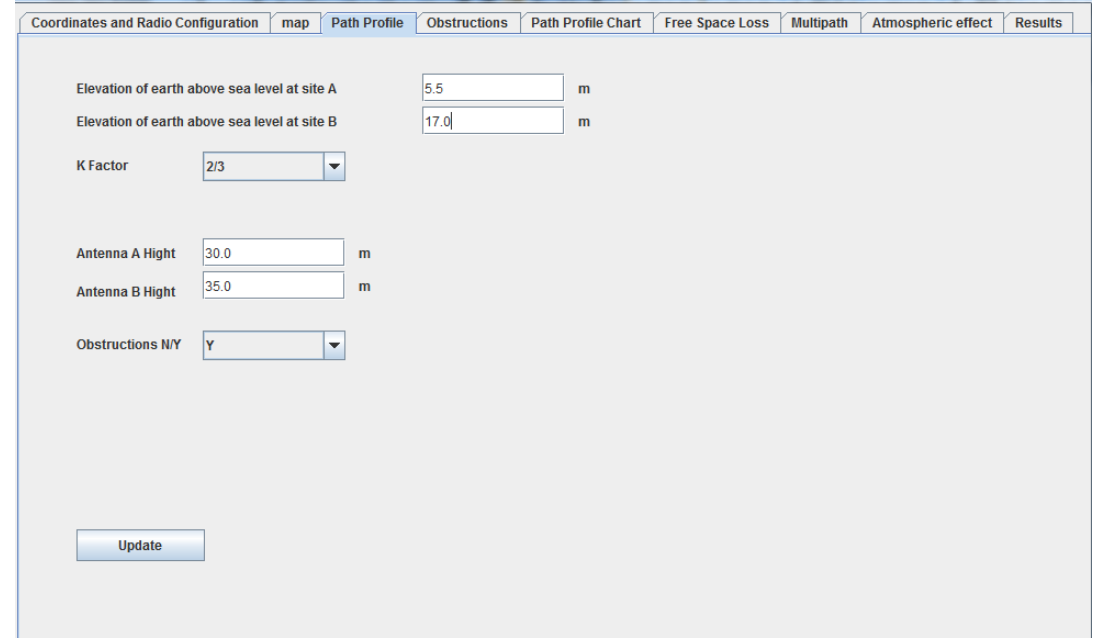

Figure 8 Path profile.

\section{4) Obstructions}

If there are any extra obstructions, the user has to add the height of each one in this part, and its name and the distance from site A for each one and the tool will calculate the highest one as in Fig. .

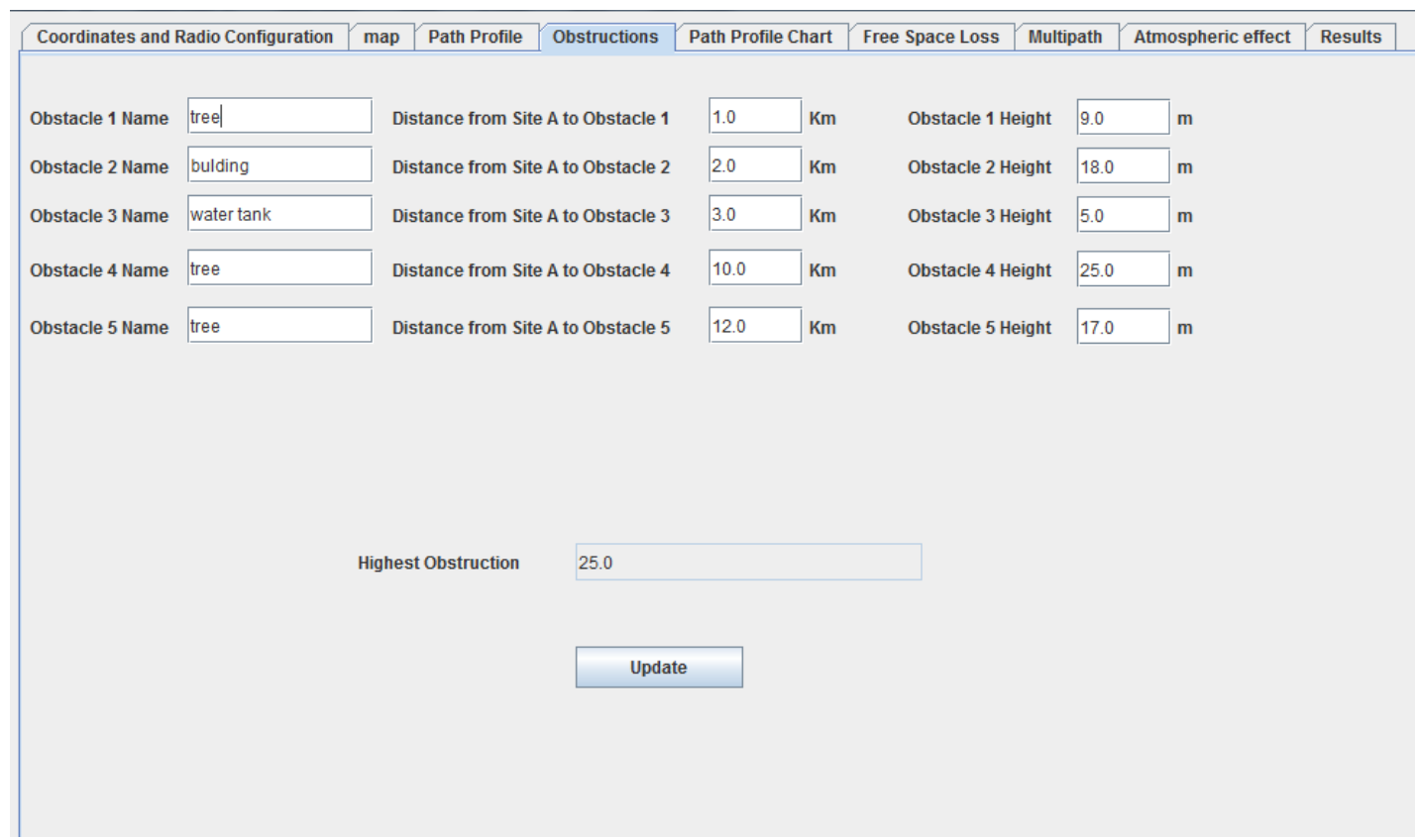

Fig. 9 Obstructions.

\section{5) Path Profile Chart}

In this part, the software draws sites A \& B, the beam with the freznel Zone, the terrain of the earth and the elevation of the obstacles. Based on the provide information, the tool shows if the line-of-sight path is clear or not Figure . 


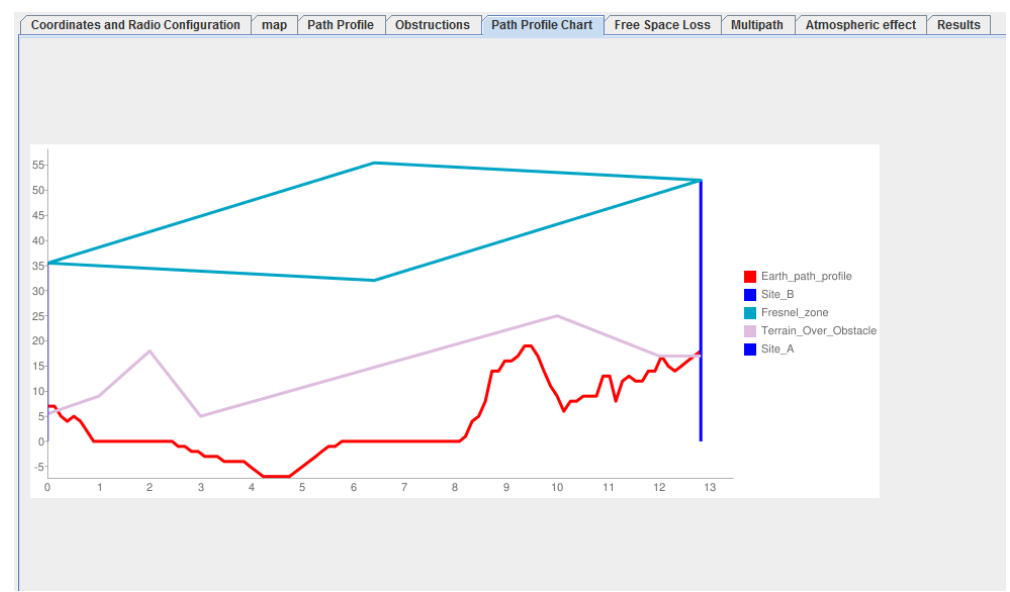

Figure 10 Path profile chart.

\section{6) Free Space Loss}

Here, the tool calculates the free space loss and the received signal level from (2). The user can put the distance to calculate the Fresnel Zone radius at it and the tool calculates it automatically from (4) as shown in Fig. .

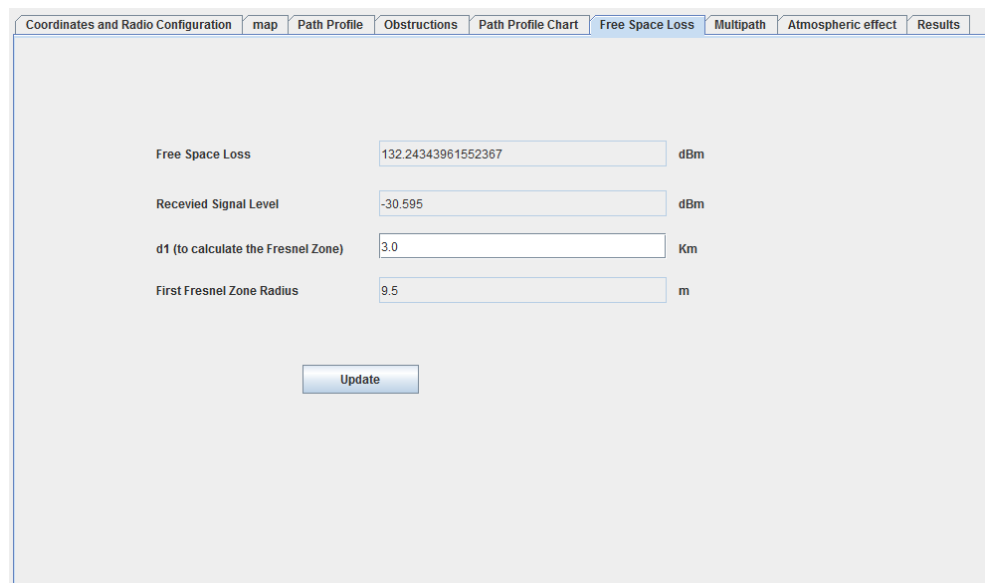

Fig. 11 Free space loss.

7) Multipath

In this part, the user chooses the Climate factor (average, dry, humid), the terrain factor (average, mountains, smooth), the natural of the terrain (Hills, Plains, mountains), the region (Europe and Africa, North and South America, Others), and the Latitude Range. If there is XPIC in the design, the user has to enter Cross polarization discrimination XPD, and Processing gain enhancement XPIF. Then, the software automatically calculates the Geoclimatic Factor (K) from (6) as shown in Fig. .

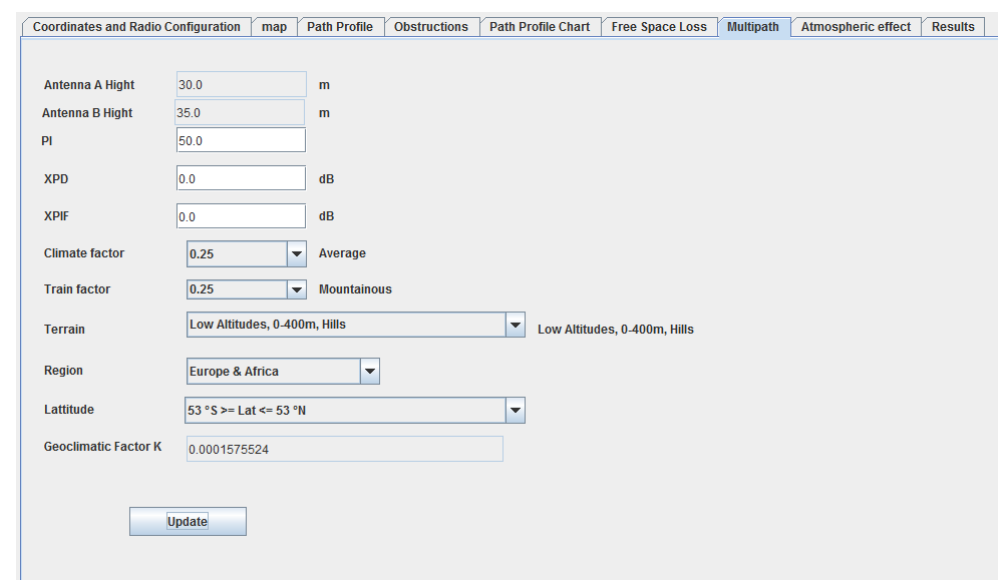

Fig. 12. Multipath. 


\section{8) Atmospheric effect}

In this part, the user chooses the rain region according to the ITU standards and enters the feeder losses, temperature, water vapor , and pressure as shown in Fig. .

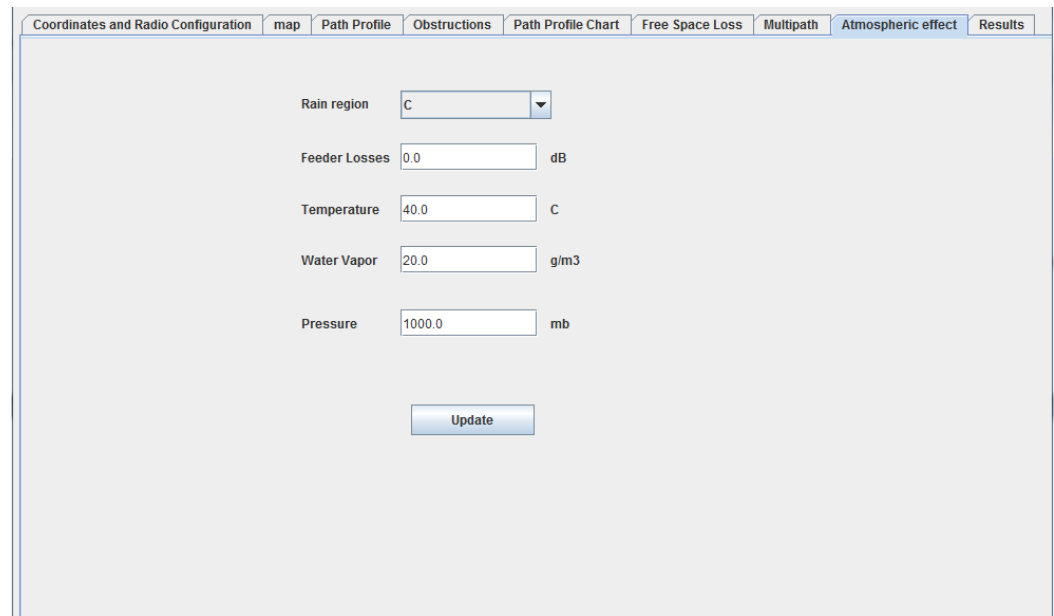

Fig. 13 .Atmospheric effect.

\section{9) Results}

Finally the results part displays all the results of this link design as illustrated in Fig. :

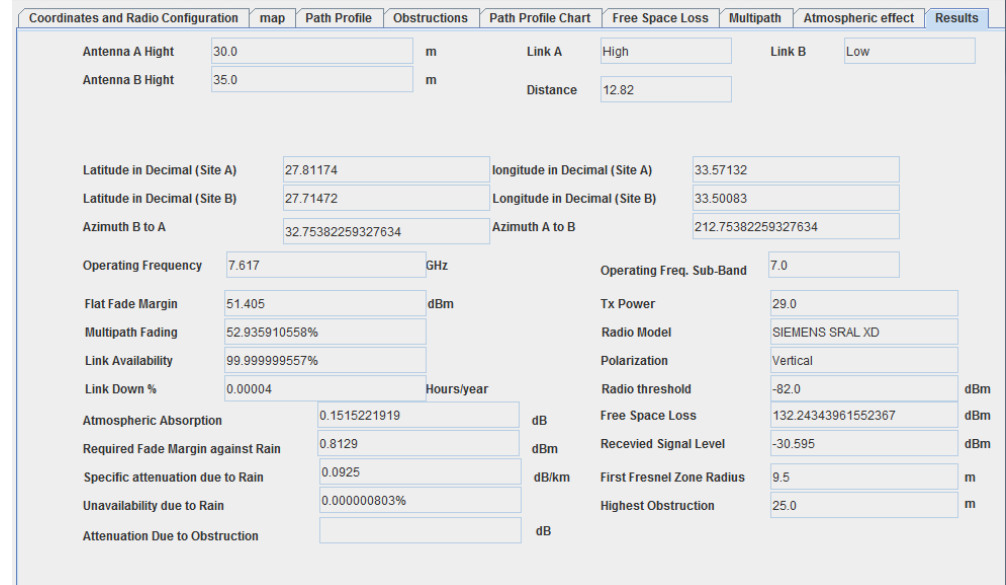

Fig. 14. Results

After defining all of the required design parameters, the implemented tool presents the results of the microwave radio link designed as following:

- $\quad$ Free space loss: it is calculated using (2). In the investigated case study, the result of the free space loss is: $132.24343961552367 \mathrm{dBm}$

- $\quad$ Flat fade margin: it is calculated according to the received signal level and the receiver threshold level for the radio equipment selected as the fade margin is equal to the difference between the received signal level and the receiver threshold level. In the investigated case study, the result of the flat fade margin is: $51.405 \mathrm{dBm}$

- Multipath fading loss: it is calculated using (5), (6), (7), (8) and according to the standards of ITU and the data entered in part 7 in the software. In the investigated case study, the result of multipath fading loss is: $52.935910558 \%$

- Link availability: it equals $1-\mathrm{P}_{\mathrm{w}}$ where:

$$
P_{w}=\left(\left[6.10^{-7} C f d^{3}\right] 10^{-\frac{F F M}{10}}\right)
$$

In the investigated case study, the result of the link availability is: $99.999999557 \%$

- $\quad$ Link down: it is the annual outage and it is calculated as follows 
$[365 * 24 * 60$ (1- link availability)]/60 Hours/year In the investigated case study, the result of the annual outage is: 0.00004 Hours/year

- $\quad$ Atmospheric absorption: it is calculated using (9), (10), (11), (12), (13) and the data entered in part 8 in the software. In the investigated case study, the result of the Atmospheric absorption is: $0.1515221919 \mathrm{~dB}$

- $\quad$ Required fade margin against rain: it is calculated using (14), (15), (16) and the standards of ITU. In the investigated case study, the result of the required fade margin against rain is: $0.8129 \mathrm{dBm}$

- $\quad$ Specific attenuation due to rain: is calculated using (14). In the investigated case study, the result of the specific attenuation due to rain is: $0.0925 \mathrm{dBm} / \mathrm{km}$

- Unavailability due to rain: it is calculated as following:

$11.628\left\{-0.546+[0.29812+0.172 \log (0.12 \text { FM due to rain } / \text { FFM })]^{1 / 2}\right\}$

In the investigated case study, the result of the unavailability due to rain is: $0.000000803 \%$

\section{PERFORMANCE CONSIDERATIONS}

This research evaluated the effect of the different parameters to show which parameters affect which outcome. So, those parameters are changed such as: obstructions effect, multipath fading, atmospheric effect and rain effect. Some of these parameters provide additional fade margins that improve the performance of the link, and some of them reflect directly in the results of the design such as: link availability, outage time and received signal level.

For example without including the atmospheric effect; the results are as following: link availability is 99.999999573, outage time is 0.00004 Hours/year, and received signal level is -30.4434

In another case with ignoring the climate factor (average, dry, humid) and the terrain factor (average, mountains, smooth); the results are as following: link availability is $100.000000000 \%$, outage time is 0.00000 Hours/year, and received signal level is -30.595

Finally, with respect to atmospheric effect, climate factor and terrain factor; the results are as following: link availability is $99.999999557 \%$, outage time is 0.00004 Hours/year, and received signal level is 30.595. Table 3 shows the change in the results in these three cases:

Table 3 Case study changes

\begin{tabular}{|l|l|l|l|}
\hline \multicolumn{1}{|c|}{ Case details } & Link availability & $\begin{array}{l}\text { Outage } \\
\text { time }\end{array}$ & $\begin{array}{l}\text { Received } \\
\text { signal level }\end{array}$ \\
\hline $\begin{array}{l}\text { Without respect to } \\
\text { atmospheric effect }\end{array}$ & $99.999999573 \%$ & 0.00004 & -30.4434 \\
\hline $\begin{array}{l}\text { Without respect to climate } \\
\text { and terrain factors }\end{array}$ & $100.000000000 \%$ & 0 & -30.595 \\
\hline $\begin{array}{l}\text { With respect to } \\
\text { atmospheric effect, } \\
\text { climate and terrain factors }\end{array}$ & $99.999999557 \%$ & 0.00004 & -30.595 \\
\hline
\end{tabular}

It is clear that there are variations in the results depending on the included and ignored parameters. So, take more parameters into consideration leads to more accurate design as shown in considered case study, which is lead to more reliable and stable microwave link. Depending on the required accuracy and complexity of the design, some parameters can be ignored.

\section{Conclusion}

The final product of this work is characterized as software that can design a new microwave radio links. The program can calculate the path length of the link between two endpoints, free space loss, flat fade margin, multipath fading loss, link availability, atmospheric absorption, and unavailability due to rain. This software is developed to help telecommunications engineers to design and simulate a new microwave line-of-sight radio links over varieties of terrain and paths such as hills, mountains, and urban areas; and under varieties atmospheric conditions without going into detailed mathematical equations. The effected of the different system paramours is studied related to the required accuracy of the design. 


\section{REFERENCES}

[1] Habeeb Khader Shankiti, "MICROWAVE COMMUNICATION SYSTEMS: TRANSMISSION CONSIDERATIONS, MEASUREMENT EQUIPMENT AND MEASURING TECHNIQVES," University Of Riyadh, 1975.

[2] Trevor Manning, "Microwave Radio Transmission Design Guide," ARTECH HOUSE,2009.

[3] Mohamed Jamil Ahmed Mulla," LONG HOP LINE-OF-SIGHT MICROWAVE LINK ACROSS THE RED SEA," University Of Riyadh, 1972.

[4] PROPAGATION DATA AND PREDICTION METHODS REQUIRED FOR THE DESIGN OF TERRESTRIAL LINE-OF-SIGHT SYSTEMS RECOMMENDATION ITU-R P.530-7.

[5] Mike O. Asiyo And Thomas J. O. Afullo , "STATISTICAL ESTIMATION OF FADE DEPTH AND OUTAGE PROBABILITY DUE TO MULTIPATH PROPAGATION IN SOUTHERN AFRICA," Progress In Electromagnetics Research B, Vol. 46, Pp. 251-274, 2013.

[6] Bassem R. Mahafza , "Radar Systems Analysis And Design Using MATLAB Third Edition," CRC Press, 2015.

[7] HOSSAIN Sakir, "Rain Attenuation Prediction For Terrestrial Microwave Link In Bangladesh," Journal Of Electrical \& Electronics Engineering;, Vol. 7 Issue 1, Pp.63-68, May2014

[8] Uzma Siddique, Laeeq Ahmad, And Gulistan Raja , "Microwave Attenuation And Prediction Of Rain Outage For Wireless Networks In Pakistan's Tropical Region," International Journal Of Microwave Science And Technology, Volume 2011, 2011.

[9] H. Abdalla Jr, Paulo Carvalho, Luis. F. Molinaro, Carlos Evangelista, L Bermudez, "TOOLS FOR MICROWAVE RADIO COMMUNICATIONS SYSTEM DESIGN," Journal Of Microwaves And Optoelectronics, Vol. 3, N.O 1, Pp.26-38, April 2003.

[10] Saad Abdulaziz Al- Bidnah, "MICROWAVE COMMUNICATION SYSTEM DESIGN IN SAUDI ARABIA," University Of Riyadh, Saudi Arabia, 1972.

[11] Samuel P. Mason, Peter S. Guest, Andreas K. Goroch, "ATMOSPHERIC EFFECTS ON RADIO FREQUENCY (RF) WAVE PROPAGATION IN A HUMID, NEAR-SURFACE ENVIRONMENT," NAVAL POSTGRADUATE SCHOOL, March 2010.

[12] Tom Garlington, "Microwave Line-Of-Sight Transmission Engineering," U.S. Army Information Systems Engineering Command (USAISEC), Transmission Systems Directorate, WP No. AMSEL-IE-TS-06015, June 2006.

[13] Characteristics Of Precipitation For Propagation Modelling, RECOMMENDATION ITU-R P.837-1, (1992-1994).

[14] Specific Attenuation Model For Rain For Use In Prediction Methods, RECOMMENDATION ITU-R P.838-3, (1992-1999-2003-2005). 